\title{
Dynamics of Bose-Einstein Condensates: Exact Representation and Topological Classification of Coherent Matter Waves
}

\author{
Leilei Jia, ${ }^{1}$ Qihuai Liu, ${ }^{2,3}$ and Shengqiang Tang ${ }^{2}$ \\ ${ }^{1}$ Department of Electrical Engineering, Guilin College of Aerospace Technology, Guilin 541004, China \\ ${ }^{2}$ School of Mathematics and Computing Science, Guilin University of Electronic Technology, Guilin 541001, China \\ ${ }^{3}$ School of Mathematical Sciences, Fudan University, Shanghai 200433, China \\ Correspondence should be addressed to Shengqiang Tang; tangsq@guet.edu.cn
}

Received 5 December 2013; Accepted 10 January 2014; Published 3 March 2014

Academic Editor: Dianchen Lu

Copyright (C) 2014 Leilei Jia et al. This is an open access article distributed under the Creative Commons Attribution License, which permits unrestricted use, distribution, and reproduction in any medium, provided the original work is properly cited.

\begin{abstract}
By using the bifurcation theory of dynamical systems, we present the exact representation and topological classification of coherent matter waves in Bose-Einstein condensates (BECs), such as solitary waves and modulate amplitude waves (MAWs). The existence and multiplicity of such waves are determined by the parameter regions selected. The results show that the characteristic of coherent matter waves can be determined by the "angular momentum" in attractive BECs while for repulsive BECs; the waves of the coherent form are all MAWs. All exact explicit parametric representations of the above waves are exhibited and numerical simulations support the result.
\end{abstract}

\section{Introduction}

Particles in a dilute gas reside in the same quantum (ground) state at low temperatures, which form a Bose-Einstein condensate (BEC). This phenomenon was first observed experimentally in 1995 with vapors of rubidium and sodium [1]. Since then the dynamics of $B E C$ have received intensive interest both in mathematics and physics, including superfluid and dissipative dynamics [2], Bloch waves [3], the dynamical stability $[4,5]$, chaos $[6,7]$, and dark and bright soliton $[8,9]$.

Considering only two-body interactions, based on the mean-field theory, the BEC is described by the Gross-Pitaevskii equation (GPE), which is a one-dimensional nonlinear Schrödinger equation [10]

$$
i \hbar \psi_{t}=-\left[\frac{\hbar^{2}}{2 m}\right] \psi_{x x}+\tilde{g}|\psi|^{2} \psi+V(x) \psi
$$

where $m$ is the mass of gas particle, $\widetilde{g}=\left[4 \pi \hbar^{2} a / m\right]\left[1+\mathcal{O}\left(\zeta^{2}\right)\right]$ and $\zeta=\sqrt{|\psi|^{2}|a|^{3}}$ are the dilute gas parameter, and $a$ is the two-body s-wave scattering length, which is determined by the atomic species in the condensate. Interactions between atoms are repulsive when $a>0$ and attractive when $a<0$.

Realizable potentials $V(x)$ of particular theoretical and experimental interest usually include harmonic traps [11], optical lattices and superlattices [12], and superpositions of lattices or superlattices with harmonic traps. However, also in many cases, weak potentials or approximately constant potentials are typically used, such as $V(x)=V_{0}+\epsilon V_{1}(x)$ with $\epsilon \ll 1$. Therefore, one can use the Lindstedts method and the multiple scale perturbation theory $[13,14]$ or the method of averaging [15-17] to study dynamics of BEC with the high order approximation. For $\epsilon=0$, the solutions of system (1) with coherent structures may be equilibrium points, standing waves, solitary waves, or even unbounded. While the small perturbed parameter $\epsilon \neq 0$, for example, Bose-Einstein condensed atoms being perturbed by a weak optical lattice potential, the dynamical characters of these solutions may be preserved $[14,15]$ as periodic or quasiperiodic modulated amplitude waves. However, under some conditions of the adjustable parameters such characters may be destroyed, and the spatial chaos in the BEC occurs [6]. Consequently, it is important to investigate the dynamics of the unperturbed 
system drastically and entirely. Some related papers we can refer to are $[18,19]$.

In this paper, we will give a full topological classification of coherent structural solutions for system (1) with various adjustable parameters, while restricting the external potentials $V(x) \equiv V_{0}$. The plane of parameters is divided into six regions. Unbounded solutions, modulated amplitude waves, and solitary waves are determined in different regions. Moreover, all exact explicit representations of solutions are exhibited in this paper. For generality, along with our analysis and derivation, we restrict the complex solutions of system (1) with nonconstant phases, which leads to nonlinear equations with singularities. Therefore, the solutions will lose smoothness in phase space. This phenomenon has been studied in other physical models by some authors (see $[20,21])$.

\section{Coherent Structures}

We consider uniformly propagating coherent structures with the ansatz

$$
\psi(x, t)=R(x) \exp (i[\theta(x)-\mu t])
$$

where $R$ gives the amplitude dynamics of the wave function with $|R(x)|=|\psi(t, x)|, \theta(x)$ gives the phase dynamics, $\mathbf{v} \propto \nabla \theta$ is the particle velocity, and $\mu$ is the BEC's chemical potential. When the (temporally periodic) coherent structure (2) is also spatially periodic, it is called periodic modulated amplitude waves (MAWs) [22]; while the coherent structure (2) is also spatially quasiperiodic, it is called quasiperiodic modulated amplitude waves (QMAWs) $[15,16]$.

Substituting (2) into the GP equation (1), and equating the real and the imaginary components of the desired equation, we obtain

$$
\begin{gathered}
\hbar \mu R(x)=-\frac{\hbar^{2} R^{\prime \prime}(x)}{2 m}+\left[\frac{\hbar\left[\theta^{\prime}(x)\right]^{2}}{2 m}+\tilde{g} R^{2}(x)+V_{0}\right] R(x), \\
0=\frac{\hbar^{2}}{2 m}\left[2 \theta^{\prime}(x) R^{\prime}(x)+\theta^{\prime \prime}(x) R(x)\right],
\end{gathered}
$$

which yields the following nonlinear Duffing equation with regularities:

$$
R^{\prime \prime}(x)=\frac{c^{2}}{R^{3}(x)}+\frac{2 m \tilde{g} R^{3}(x)}{\hbar^{2}}+\frac{2 m}{\hbar^{2}}\left(V_{0}-\mu \hbar\right) R(x) .
$$

The parameter $c$ is given by the relation

$$
\theta^{\prime}(x)=\frac{c}{R^{2}(x)}
$$

which indicates conservation of angular momentum [23]. Null angular momentum solutions satisfying $c=0$ are standing waves of system (1), which have been described in detail [14]. Here, we restrict that the parameter $c$ is nonzero.To the best of our knowledge, we have not seen any overall classification of solutions in this case. Consequently, (4) becomes

$$
R^{\prime \prime}+\delta R-\frac{c^{2}}{R^{3}}+g R^{3}=0,
$$

where $\delta=2 m\left(\mu \hbar-V_{0}\right) / \hbar^{2}$ and $g=2 m \tilde{g} / \hbar^{2}$. Consequently, (6) is equivalent to the two-dimensional system

$$
\frac{\mathrm{d} R}{\mathrm{~d} x}=S, \quad \frac{\mathrm{d} S}{\mathrm{~d} x}=\frac{c^{2}-\delta R^{4}-g R^{6}}{R^{3}}
$$

with the first integral

$$
H(R, S)=S^{2}+\frac{c^{2}+\delta R^{4}+R^{6} g / 2}{R^{2}}=h .
$$

\section{Bifurcations of Phase Portraits}

The spatial dynamics of amplitudes $R(x)$ for the wave functions in BECs are determined by nonlinear system (7), which depends on the parameter variables $c, g, \delta$. The parameter $\delta$ is related to the chemical potential $\mu$ and the external potential $V_{0}$, and $g$ has the same sign of the scatter length a. The parameter $c$ comes from the constant of integration, playing the role of "angular momentum". Without loss of generality, we assume that $c>0$.

For a fixed positive constant $c$, the characteristics of the orbits in the phase plane $(R, S)$ are affected by two parameters $g, \delta$. Consequently, in the following we shall give an overfull classification of the orbits by the regions selecting for $(g, \delta)$. The bifurcations of phase portraits will be also investigated.

Let $d x=R^{3} d \zeta$. Then, excepting the straight line $R=0$, the system (7) has the same topological phase portraits as the following system:

$$
\frac{\mathrm{d} R}{\mathrm{~d} \zeta}=R^{3} S, \quad \frac{\mathrm{d} S}{\mathrm{~d} \zeta}=c^{2}-\delta R^{4}-g R^{6} .
$$

Now, the straight line $R=0$ is an integral invariant straight line of (9). Denote that $f(R)=c^{2}-\delta R^{4}-g R^{6}$, then we have $f^{\prime}(R)=-2 R^{3}\left(2 \delta+3 g R^{2}\right)$. To find equilibrium points of (9) which are corresponding to the constant-amplitude waves, we have to solve the following algebraic equation:

$$
f(R)=c^{2}-\delta R^{4}-g R^{6}=0 .
$$

Note that when $R=R_{ \pm}= \pm \sqrt{-2 \delta / 3 g}, f^{\prime}\left(R_{ \pm}\right)=0$. Thus, we have $f\left(R_{ \pm}\right)=c^{2}-\delta R_{ \pm}^{4}-g R_{ \pm}^{6}$, which implies the relations between $\delta$ and $3(\mathrm{cg} / 2)^{2 / 3}$ in the $(g, \delta$-) parameter plane determining the existence and multiplicity of equilibrium points. An example of the number of roots depending on the sign of $3(\mathrm{cg} / 2)^{2 / 3}-\delta$ is shown in Figure 1 .

In the $(g, \delta$-) parameter plane, both the curve $L: \delta=$ $3(\mathrm{cg} / 2)^{2 / 3}$ and the straight line $g=0$ partition the plane into six regions. Thus, we have to take six cases to be considered.

(i) If $\delta=3(\mathrm{cg} / 2)^{2 / 3}, g>0$, there are two equilibrium points of (9):

$$
A_{1}\left(-\frac{3 c}{2 \delta} \sqrt{g}, 0\right), \quad A_{2}\left(\frac{3 c}{2 \delta} \sqrt{g}, 0\right) .
$$


(ii) If $\delta=3(\mathrm{cg} / 2)^{2 / 3}, g<0$, there are two equilibrium points of (9):

$$
B_{1}\left(-\frac{3 c}{2 \delta} \sqrt{-2 g}, 0\right), \quad B_{2}\left(\frac{3 c}{2 \delta} \sqrt{-2 g}, 0\right) .
$$

(iii) If $\delta<3(\mathrm{cg} / 2)^{2 / 3}, g>0$, there are two equilibrium points of (9):

$$
C_{1}\left(-\sqrt{\widetilde{R}-\frac{\delta}{3 g}}, 0\right), \quad C_{2}\left(\sqrt{\widetilde{R}-\frac{\delta}{3 g}}, 0\right),
$$

where

$$
\begin{gathered}
\widetilde{R}=\left(-\frac{q}{2}+\frac{\sqrt{\Delta}}{2}\right)^{1 / 3}+\left(-\frac{q}{2}-\frac{\sqrt{\Delta}}{2}\right)^{1 / 3}, \\
q=\frac{1}{g}\left(-c^{2}+\frac{2 \delta^{3}}{27 g^{2}}\right), \quad \Delta=\frac{c^{2}\left(27 c^{2} g^{2}-4 \delta^{3}\right)}{27 g^{4}} .
\end{gathered}
$$

(iv) If $\delta>3(\mathrm{cg} / 2)^{2 / 3}, g>0$, there are two equilibrium points of (9):

$$
\begin{aligned}
& D_{1}\left(-\sqrt{2(r)^{1 / 3} \cos \frac{\phi}{3}-\frac{\delta}{3 g}}, 0\right), \\
& D_{2}\left(\sqrt{2(r)^{1 / 3} \cos \frac{\phi}{3}-\frac{\delta}{3 g}}, 0\right),
\end{aligned}
$$

where

$$
r=\frac{\delta^{3}}{27 g^{3}}, \quad \cos \phi=\frac{c^{2}-2 \delta^{3} / 27 g^{2}}{2 g r}, \quad \sin \phi=\frac{\sqrt{-\Delta}}{r} .
$$

(v) If $\delta>3(\mathrm{cg} / 2)^{2 / 3}, g<0$, there are four equilibrium points of (9):

$$
E_{1}\left(-R_{1}, 0\right), \quad E_{2}\left(-R_{2}, 0\right), \quad E_{3}\left(R_{1}, 0\right), \quad E_{4}\left(R_{2}, 0\right),
$$

where $R_{1}>R_{2}$ satisfies

$$
\begin{aligned}
& R_{1}=\sqrt{2(r)^{1 / 3} \cos \frac{\phi+2 \pi}{3}-\frac{\delta}{3 g}}, \\
& R_{2}=\sqrt{2(r)^{1 / 3} \cos \frac{\phi+4 \pi}{3}-\frac{\delta}{3 g}} .
\end{aligned}
$$

(vi) If $\delta<3(\mathrm{cg} / 2)^{2 / 3}, \mathrm{~g}<0$, there are no equilibrium points of (9).

Let $M\left(R_{e}, S_{e}\right)$ be the coefficient matrix of the linearized system of (9) at an equilibrium point $\left(R_{e}, y_{e}\right)$. Then, we have

$$
J\left(R_{e}, 0\right)=\operatorname{det}\left(M\left(R_{e}, 0\right)\right)=-2 R_{e}^{6}\left(2 \delta+3 g R_{e}^{2}\right) .
$$

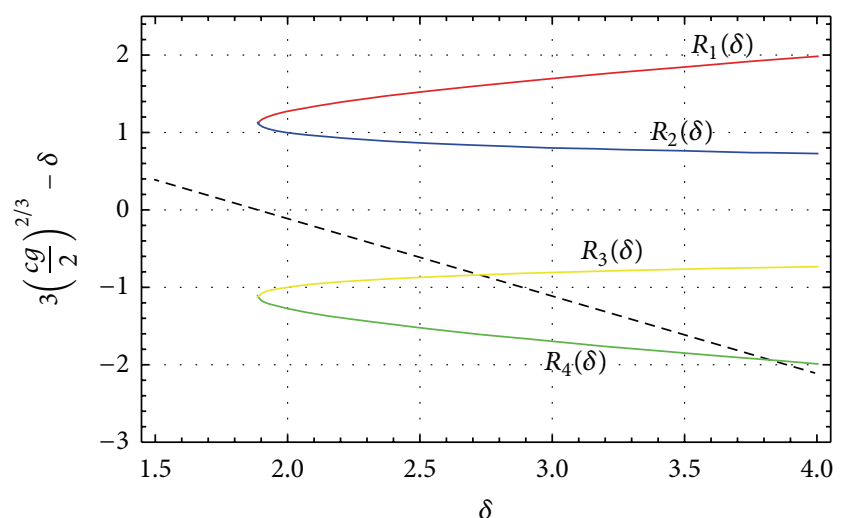

Figure 1: An example of the number of roots for $f(R)$ depending on the sign of $3(\mathrm{cg} / 2)^{2 / 3}-\delta$ with $c=1, g=-1$. There are 4 roots for $\delta>1.8899,2$ roots for the threshold $\delta=1.8899$, and no root for $\delta<1.8899$.

TABLE 1: Type of equilibria of (7).

\begin{tabular}{lcc}
\hline Regions of $(g, \delta)$ & Equilibria & Type \\
\hline (I): $\delta=3\left(\frac{c g}{2}\right)^{2 / 3}, g>0$ & $A_{1}, A_{2}$ & Center, center \\
(II): $\delta=3\left(\frac{c g}{2}\right)^{2 / 3}, g<0$ & $B_{1}, B_{2}$ & Saddle, saddle \\
(III): $\delta<3\left(\frac{c g}{2}\right)^{2 / 3}, g>0$ & $C_{1}, C_{2}$ & Center, center \\
(IV): $\delta>3\left(\frac{c g}{2}\right)^{2 / 3}, g>0$ & $D_{1}, D_{2}$ & Center, center \\
$(\mathrm{V}): \delta>3\left(\frac{c g}{2}\right)^{2 / 3}, g<0$ & $E_{1}, E_{2}$ & Saddle, center \\
(VI): $\delta<3\left(\frac{c g}{2}\right)^{2 / 3}, g<0$ & $E_{3}, E_{4}$ & Center, saddle \\
\hline
\end{tabular}

By the theory of planar dynamical systems, we know that for an equilibrium point of a planar integrable system if $J<0$, then the equilibrium point is a saddle point; if $J>0$ and $\operatorname{Trace}\left(M\left(R_{e}, y_{e}\right)\right)=0$, then it is a center point; if $J>0$ and $\left(\operatorname{Trace}\left(M\left(R_{e}, y_{e}\right)\right)\right)^{2}-4 J\left(R_{e}, y_{e}\right)>0$, then it is a node; if $J=0$ and the index of the equilibrium point is 0 , then it is a cusp; otherwise, it is a high order equilibrium point.

For the function defined by (8), we denote that

$$
h_{i}=H\left( \pm R_{i}, 0\right)=\frac{c^{2}+\delta R_{i}^{4}+(g / 2) R_{i}^{6}}{R_{i}^{2}}, \quad i=1,2 .
$$

We next use the above statements to consider the bifurcations of the phase portraits of (7). The possible qualitative dynamics (for $c \neq 0$ ) are illustrated in Figure 2 and summarized in Table 1 .

\section{Exact Explicit Representations of Matter Waves}

In this section, we give all exact explicit parametric representations of solitary wave solutions and periodic wave solutions (MAWs or QMAWs). Denote $\operatorname{sn}(v, k)$ the Jacobian elliptic 


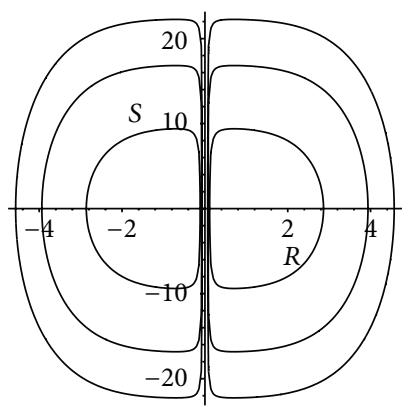

(a) $(g, \delta) \in(\mathrm{I})$

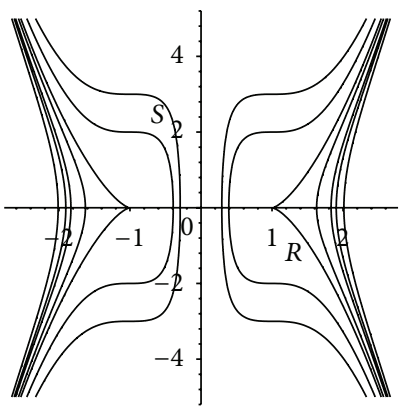

(b) $(g, \delta) \in(\mathrm{II})$

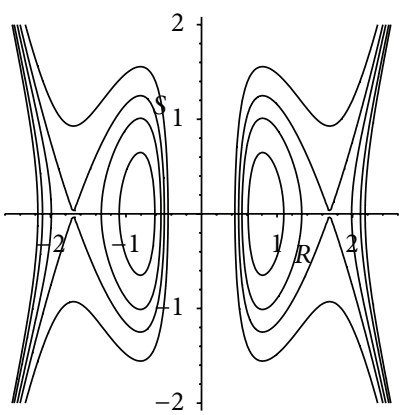

(e) $(g, \delta) \in(\mathrm{V})$

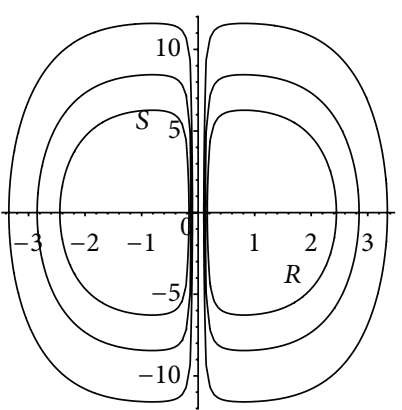

(c) $(g, \delta) \in(\mathrm{III})$

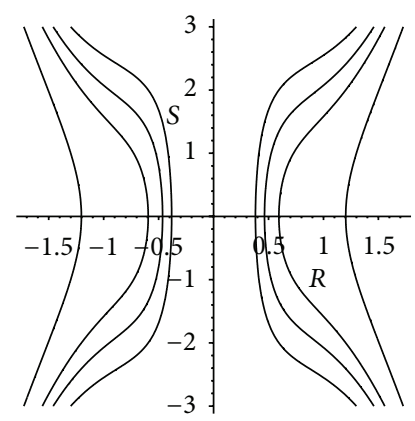

(f) $(g, \delta) \in(\mathrm{VI})$

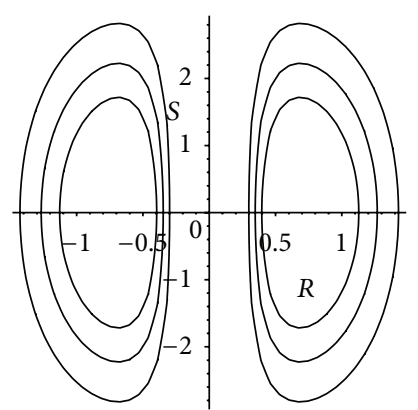

(d) $(g, \delta) \in(I V)$

FIGURE 2: The phase portraits of (7).

function with the modulus $k$ and $\prod\left(\varphi, \alpha^{2}, k\right)$ the Legendre incomplete elliptic integral of the third kind (see [24]). Two types of BECs will be considered, respectively.

4.1. Attractive BECs $(g<0)$. (a) Suppose that $(g, \delta) \in(\mathrm{V})$; that is, $g<0, c>0, \delta>3(\mathrm{cg} / 2)^{2 / 3}$. In this case, we have the phase portrait of (7) as shown in Figure 2(e).

(1) Notice that $H\left( \pm R_{1}, 0\right)=h_{1}$ with $R_{1}>0$, corresponding to the curve defined by $H(R, S)=h_{1}$, and there is a homoclinic orbit connecting the equilibrium point $E_{1}\left(R_{1}, 0\right)$ or $E_{4}\left(-R_{1}, 0\right)$. The arch curve has the algebraic equation

$$
\begin{aligned}
S^{2} & =\frac{h_{1} R^{2}-\left(c^{2}+\delta R^{4}+(g / 2) R^{6}\right)}{R^{2}} \\
& =-\frac{g}{2 R^{2}}\left(R^{2}-R_{1}^{2}\right)^{2}\left(R^{2}+\frac{2 c^{2}}{g R_{1}^{4}}\right) .
\end{aligned}
$$

By using the first equation of (7), for (6), we obtain the parametric representation of a smooth solitary wave solution of valley type and a smooth solitary wave solution of peak type as follows:

$$
\begin{aligned}
R(x) & = \pm \sqrt{-\frac{2 c^{2}}{g R_{1}^{4}}+\left(R_{1}^{2}+\frac{2 c^{2}}{g R_{1}^{4}}\right) \tanh ^{2}\left(\frac{\Omega_{1}^{1 / 2} x}{R_{1}}\right)} \\
& = \pm \frac{1}{R_{1}} \sqrt{R_{1}^{4}+\frac{2 \Omega_{1}}{g}\left[1-\tanh ^{2}\left(\frac{\Omega_{1}^{1 / 2} x}{R_{1}}\right)\right]},
\end{aligned}
$$

where $\Omega_{1}=\delta R_{1}^{2}-h_{1}$.
Thus, by integrating (5) on both sides we have

$$
\begin{aligned}
\theta(x) & =\int \frac{c g R_{1}^{2} \mathrm{~d} x}{g R_{1}^{4}+2 \Omega_{1}\left[1-\tanh ^{2}\left(\Omega_{1}^{1 / 2} x / R_{1}\right)\right]} \\
& =\frac{x}{R_{1}^{2}}+\frac{2 \Omega_{1}}{R_{1}^{2}} \int \frac{\mathrm{d} x}{2 \Omega_{1}+g R_{1}^{4} \cosh ^{2}\left(\Omega_{1}^{1 / 2} x / R_{1}\right)} \\
& =\frac{x}{R_{1}^{2}}+\frac{2 \Omega_{1}^{1 / 4}}{R_{1} \sqrt{-\Omega_{2}}} \arctan \frac{g R_{1}^{4}\left(e^{\left(2 \sqrt{\Omega_{1}} / R_{1}\right) x}-1\right)+\Omega_{2}}{-2 \sqrt{-\Omega_{1} \sqrt{\Omega_{2}}}},
\end{aligned}
$$

where

$$
\Omega_{1}=\delta R_{1}^{2}-h_{1}, \quad \Omega_{2}=2\left(g R_{1}^{4}+2 \sqrt{\Omega_{1}}\right) .
$$

Therefore, system (1) has the following solution:

$$
\psi(t, x)=R(x) \exp [i(\theta(x)-\mu t)]
$$

with

$$
\begin{gathered}
R(x)= \pm \frac{1}{R_{1}} \sqrt{\frac{1}{g}\left(g R_{1}^{4}+2 \Omega_{1}-2 \Omega_{1} \tanh ^{2}[\phi(x)]\right)}, \\
\theta(x)=\frac{x}{R_{1}^{2}}+\frac{2 \Omega_{1}^{1 / 4}}{R_{1} \sqrt{-\Omega_{2}}} \arctan \frac{g R_{1}^{4}\left(e^{\left(2 \sqrt{\left.\Omega_{1} / R_{1}\right) x}-1\right)+\Omega_{2}}\right.}{-2 \sqrt{-\Omega_{1} \sqrt{\Omega_{2}}}} .
\end{gathered}
$$




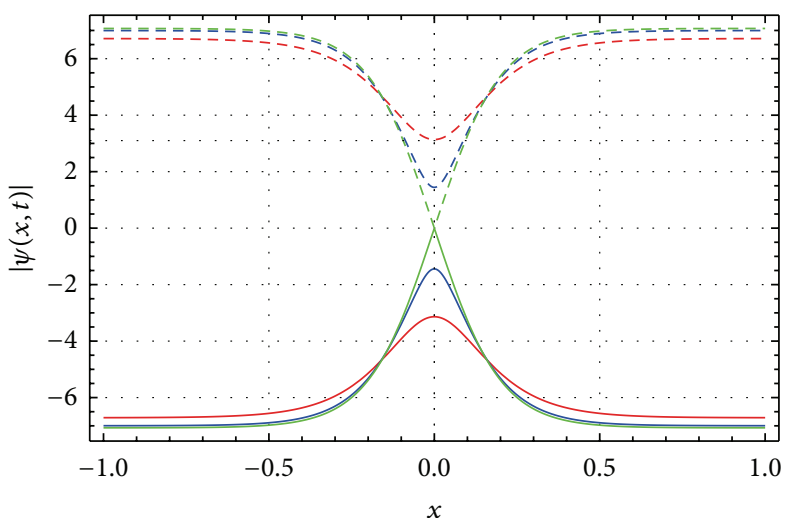

$$
\begin{array}{ll}
\text { Dark solitons } & \text { Bright solitons } \\
---c=100 & -c=100 \\
---c=50 & -c=50 \\
--c=1 & -c=1
\end{array}
$$

(a) The portrait of the amplitudes

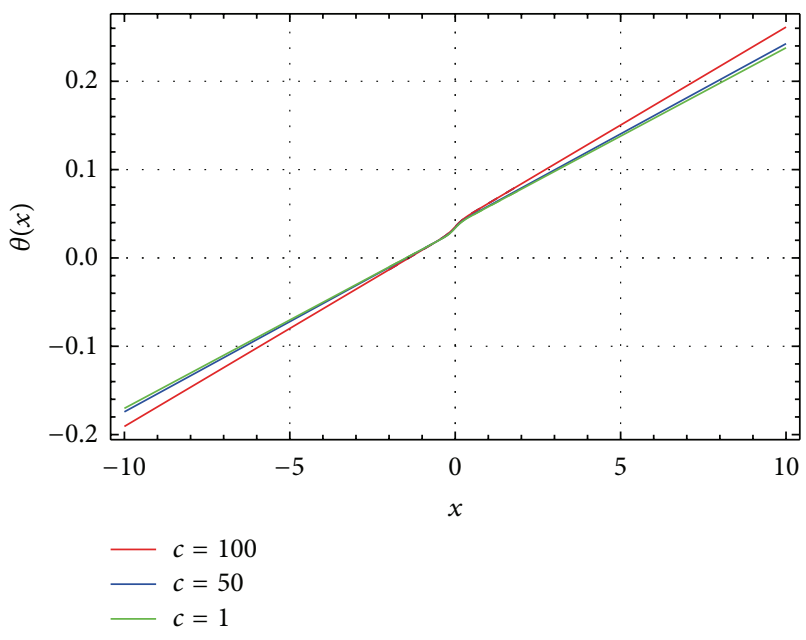

(b) The portrait of the phases

Figure 3: Dynamical evolution of the dark and bright solitons for an attractive condensate with $g=-1, \delta=50$. With the increasing of $c$ ("angular momentum"), the height of solitons decreases. The phase is monotonously increasing with respect to the spatial $x$.

(2) Recall that $h_{2}=H\left( \pm R_{2}, 0\right)$ with the equilibriums $E_{2}\left(R_{2}, 0\right), E_{3}\left(-R_{2}, 0\right)$, where the function $H(R, S)$ is defined by (8). For each $h \in\left(h_{2}, h_{1}\right), H(R, S)=h$ defines two families of periodic orbits of system (7) enclosing the center $\left(R_{2}, 0\right)$ or $\left(-R_{2}, 0\right)$, which lie on both sides of the straight line $R=0$. Those orbits determine infinitely many (positive measure set) periodic wave solutions of system (1). Using factorization of polynomial, the arch curve has the algebraic equation

$$
\begin{aligned}
S^{2} & =-\frac{g}{2 R^{2}}\left(R^{6}+\frac{2 \delta}{g} R^{4}-\frac{2 h}{g} R^{2}+\frac{2 c^{2}}{g}\right) \\
& =-\frac{g}{2 R^{2}}\left(R^{2}-R_{M}\right)\left(R^{2}-R_{m}\right)\left(R^{2}-R_{l}\right),
\end{aligned}
$$

where $0<R_{m}<R_{l}<R_{M}$. From (7), we obtain the parametric representation of the periodic wave solutions as follows:

$$
R(x)= \pm \sqrt{R_{m}+\left(R_{l}-R_{m}\right) \mathrm{sn}^{2}\left(U_{M m} x, V_{M m}\right)},
$$

where

$$
U_{M m}=\frac{1}{2} \sqrt{-2 g\left(R_{M}-R_{m}\right)}, \quad V_{M m}=\sqrt{\frac{R_{l}-R_{m}}{R_{M}-R_{m}}} .
$$

The least positive period $T$ of $R(x)$ is given by

$$
T=\frac{2}{\pi} \int_{0}^{\pi / 2} \frac{\mathrm{d} t}{\sqrt{1-V_{M m}^{2} \sin ^{2} t}} .
$$

Similarly, we have

$$
\begin{aligned}
\theta(x) & =\int \frac{c \mathrm{~d} x}{R_{m}+\left(R_{l}-R_{m}\right) \mathrm{sn}^{2}\left(U_{M m} x, V_{M m}\right)} \\
& =\Pi\left(\varphi, \alpha^{2}, \sqrt{\frac{R_{l}-R_{m}}{R_{M}-R_{m}}}\right),
\end{aligned}
$$

where $\alpha^{2}=R_{m}-R_{l} / R_{m}$.
Consequently, system (1) has the following solution of the MAW:

$$
\psi(t, x)=R(x) \exp [i(\theta(x)-\mu t)]
$$

with

$$
\begin{gathered}
R(x)= \pm \sqrt{R_{m}+\left(R_{l}-R_{m}\right) \operatorname{sn}^{2}\left(U_{M m} x, V_{M m}\right)}, \\
\theta(x)=\Pi\left(\varphi, \alpha^{2}, \sqrt{\frac{R_{l}-R_{m}}{R_{M}-R_{m}}}\right) .
\end{gathered}
$$

(b) Suppose that $(g, \delta) \in(\mathrm{II})$ or (VI). In this situation, all solutions of (6) are unbounded except two equilibriums $B_{1}$, $B_{2}$ in case of the parameters belonging to the region (II).

4.2. Repulsive BECs $(g>0)$. For repulsive BECs, the phase portraits of amplitude equation (6) have been shown in Figures 2(a), 2(c), and 2(d), respectively. The equivalent system (7) always has two center equilibriums $\left( \pm R_{*}, 0\right)$. Let $h_{*}=$ $H\left(R_{*}, 0\right)$. For each $h \in\left(h_{*},+\infty\right)$, corresponding to $H(R, S)=$ $h$, system (6) have two families of periodic orbits enclosing the two centers, respectively. All these periodic orbits, filling the whole plane except for the line $R=0$, determine infinitely many periodic wave solutions of (1). Similar to Section 4.1, system (1) have the parametric representation of these arches as (28) and (31) by the coherent form (2). In this case, none of solitary waves of the coherent form can exist while the external potential $V_{0}$ is constant.

\section{Numerical Simulations}

In order to explain the previous results better, we perform numerical simulations for solutions of solitary waves and MAWs. We emphasize the best complex case; that is, $(\delta, g) \in$ 


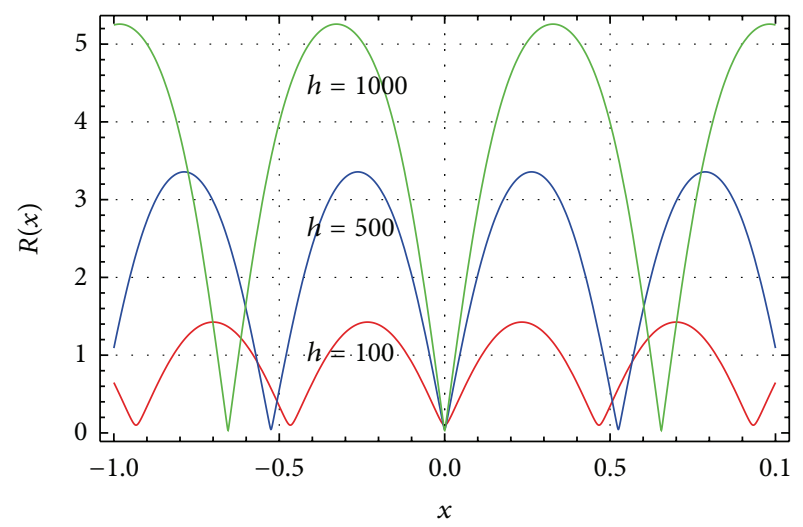

(a) The portrait of the amplitudes

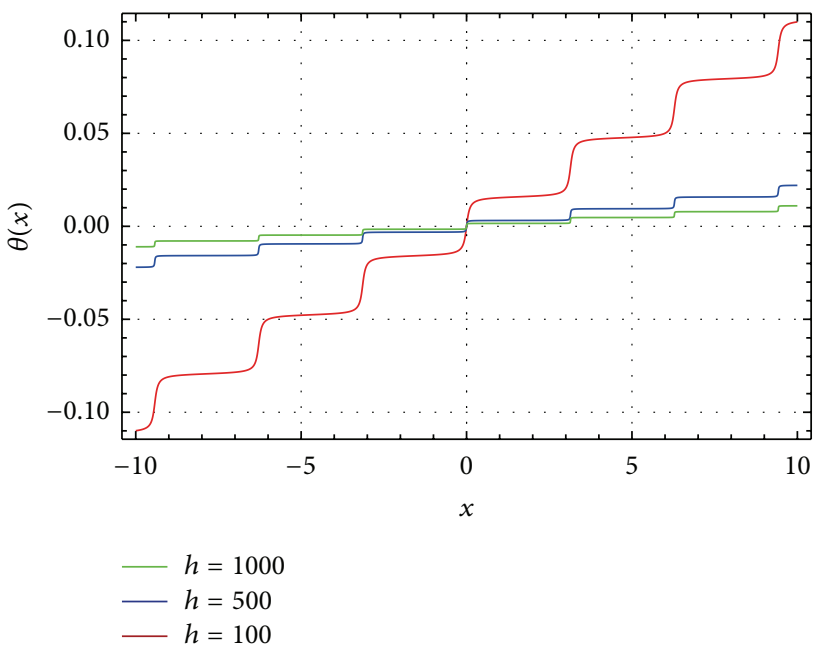

(b) The portrait of the phases

FIGURE 4: Dynamical evolution of MAWs for an attractive condensate with $g=-1, \delta=50, c=1$. For each $h \in(14.1321,1250.02)$, the curve defined by (8) in the phase space is corresponding to a MAW. The period $T(h)$ of amplitude $R$ is increasing with $h$.

(V), and other cases can be dealt with similarly. Now let us fix the parameters $g=-1, \delta=50$ which are corresponding to the attractive interactions in BECs.

While taking the "angular momentum" $c \in(0,136.0828)$, we have four equilibriums for system (7). For example, when taking the constant of integration $c=100$, we have $E_{1}:(-6.71409,0), E_{2}:(-4.19004,0), E_{3}:(4.19004,0)$, and $E_{4}:(6.71409,0)$. As shown in Figure $2(\mathrm{e})$, there are two homoclinic orbits lying in the intersection of the stable manifold and the unstable manifold of the equilibriums $E_{1}$ and $E_{4}$, respectively. More precisely, there exist two solutions $\left(R_{i}(x), S_{i}(x)\right), i=1,4$ such that

$$
\left(R_{i}(x), S_{i}(x)\right) \longrightarrow E_{i}, \quad \text { as } x \longrightarrow \pm \infty, i=1,4 .
$$

For each fixed "angular momentum" $c \in(0,136.0828)$, near $E_{1}$ the homoclinic orbit is corresponding to a dark soliton, while near $E_{4}$ the homoclinic orbit is corresponding to a bright soliton. The height of solitons, $\sup R(x ; c)-$ $\inf R(x ; c)$, is varying as the value of $c$ and the phase $\theta(x)$ is also monotonous increasing with respect to the spatial $x$, which can be shown in Figure 3 .

Also, for each fixed $c \in(0,136.0828)$, near the equilibriums $E_{2}$ and $E_{3}$, there are infinitely many periodic orbits of (7) as shown in Figure 4.

While the constant $c$ exceeds or arrives at the threshold $c=136.0828$, all those periodic orbits or solitons are destroyed and become unbounded. This indicates that the characteristic of coherent matter waves can be determined by the "angular momentum".

\section{Conflict of Interests}

The authors declare that there is no conflict of interests regarding the publication of this paper.

\section{Acknowledgment}

This work is supported by the National Natural Science Foundation of China (Grants nos. 11301106, 11226130, 11361017, and 11261013).

\section{References}

[1] K. B. Davis, M.-O. Mewes, M. R. Andrews et al., "Bose-Einstein condensation in a gas of sodium atoms," Physical Review Letters, vol. 75, no. 22, pp. 3969-3973, 1995.

[2] S. Burger, F. S. Cataliotti, C. Fort et al., "Superfluid and dissipative dynamics of a Bose-Einstein condensate in a periodic optical potential," Physical Review Letters, vol. 86, no. 20, pp. 4447-4450, 2001.

[3] B. Wu, R. B. Diener, and Q. Niu, "Bloch waves and bloch bands of Bose-Einstein condensates in optical lattices," Physical Review A, vol. 65, no. 2, Article ID 025601, 4 pages, 2002.

[4] A. Chen, S. Wen, and W. Huang, "Existence and orbital stability of periodic wave solutions for the nonlinear Schrödinger equation," The Journal of Applied Analysis and Computation, vol. 2, no. 2, pp. 137-148, 2012.

[5] B. Deconinck, B. A. Frigyik, and J. N. Kutz, "Dynamics and stability of Bose-Einstein condensates: the nonlinear Schrödinger equation with periodic potential," Journal of Nonlinear Science, vol. 12, no. 3, pp. 169-205, 2002.

[6] G. Chong, W. Hai, and Q. Xie, "Spatial chaos of trapped BoseEinstein condensate in one-dimensional weak optical lattice potential," Chaos, vol. 14, no. 2, pp. 217-223, 2004.

[7] G. Chong, W. Hai, and Q. Xie, "Controlling chaos in a weakly coupled array of Bose-Einstein condensates," Physical Review E, vol. 71, no. 1, Article ID 016202, 2005.

[8] P. Dyke, S. Lei, and R. Hulet, "Phase-dependent Interactions of Bright Matter-Wave Solitons," Bulletin of the American Physical Society, vol. 57, no. 5, Article ID U6. 00001, 2012.

[9] Y. Xu, Y. Zhang, and B. Wu, "Bright solitons in spin-orbitcoupled Bose-Einstein condensates," Physical Review A, vol. 87, no. 1, Article ID 013614, 2013. 
[10] B. Schlein and H. T. Yau, "Derivation of the Gross-Pitaevskii equation for the dynamics of Bose-Einstein condensate," Annals of Mathematics, vol. 172, no. 1, pp. 291-370, 2010.

[11] W. Krauth, "Quantum Monte Carlo calculations for a large number of bosons in a harmonic trap," Physical Review Letters, vol. 77, no. 18, pp. 3695-3699, 1996.

[12] S. Nascimbène, Y. Chen, M. Atala et al., "Experimental realization of plaquette resonating valence-bond states with ultracold atoms in optical superlattices," Physical Review Letters, vol. 108, no. 20, Article ID 205301, 2012.

[13] V. P. Chua and M. A. Porter, "Spatial resonance overlap in BoseEinstein condensates in superlattice potentials," International Journal of Bifurcation and Chaos, vol. 16, no. 4, pp. 945-959, 2006.

[14] M. A. Porter and P. G. Kevrekidis, "Bose-Einstein condensates in super-lattices," SIAM Journal on Applied Dynamical Systems, vol. 4, no. 4, pp. 783-807, 2005.

[15] Q. Liu and D. Qian, "Modulated amplitude waves with nonzero phases in Bose-Einstein condensates," Journal of Mathematical Physics, vol. 52, no. 8, Article ID 082702, 11 pages, 2011.

[16] Q. Liu and D. Qian, "Construction of modulated amplitude waves via averaging in collisionally inhomogeneous Bose-Einstein condensates," Journal of Nonlinear Mathematical Physics, vol. 19, no. 2, Article ID 1250017, 14 pages, 2012.

[17] M. A. Porter, P. G. Kevrekidis, and B. A. Malomed, "Resonant and non-resonant modulated amplitude waves for binary BoseEinstein condensates in optical lattices," Physica D, vol. 196, no. 1-2, pp. 106-123, 2004.

[18] X. Li, J. Han, and F. Wang, "The extended Riccati equation method for travelling wave solutions of $\mathrm{ZK}$ equation," The Journal of Applied Analysis and Computation, vol. 2, no. 4, pp. 423-430, 2012.

[19] S. Chow, M. Jiang, and X. Lin, "Traveling wave solutions in coupled Chua's circuits, part I: periodic solutions," The Journal of Applied Analysis and Computation, vol. 3, no. 3, pp. 213-237, 2013.

[20] J. Li and Z. Liu, "Traveling wave solutions for a class of nonlinear dispersive equations," Chinese Annals of Mathematics. Series B, vol. 23, no. 3, pp. 397-418, 2002.

[21] J. Li and G. Chen, "On a class of singular nonlinear traveling wave equations," International Journal of Bifurcation and Chaos, vol. 17, no. 11, pp. 4049-4065, 2007.

[22] L. Brusch, A. Torcini, M. van Hecke, M. G. Zimmermann, and M. Bär, "Modulated amplitude waves and defect formation in the one-dimensional complex Ginzburg-Landau equation," Physica D, vol. 160, no. 3-4, pp. 127-148, 2001.

[23] J. C. Bronski, L. D. Carr, B. Deconinck, and J. N. Kutz, "BoseEinstein condensates in standing waves: the cubic nonlinear Schrödinger equation with a periodic potential," Physical Review Letters, vol. 86, no. 8, pp. 1402-1405, 2001.

[24] P. F. Byrd and M. D. Friedman, Handbook of Elliptic Integrals for Engineers and Scientists, vol. 67 of Die Grundlehren der mathematischen Wissenschaften, Springer, New York, NY, USA, 2nd edition, 1971. 


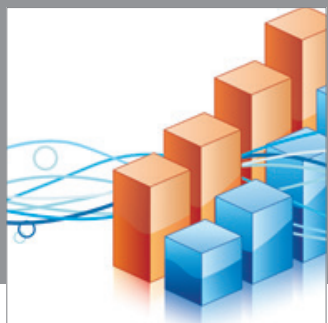

Advances in

Operations Research

mansans

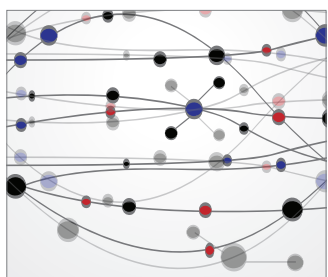

The Scientific World Journal
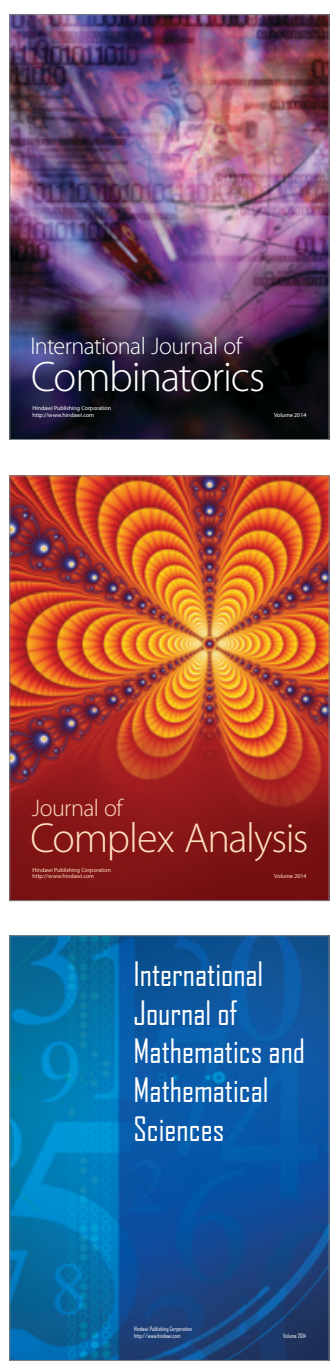
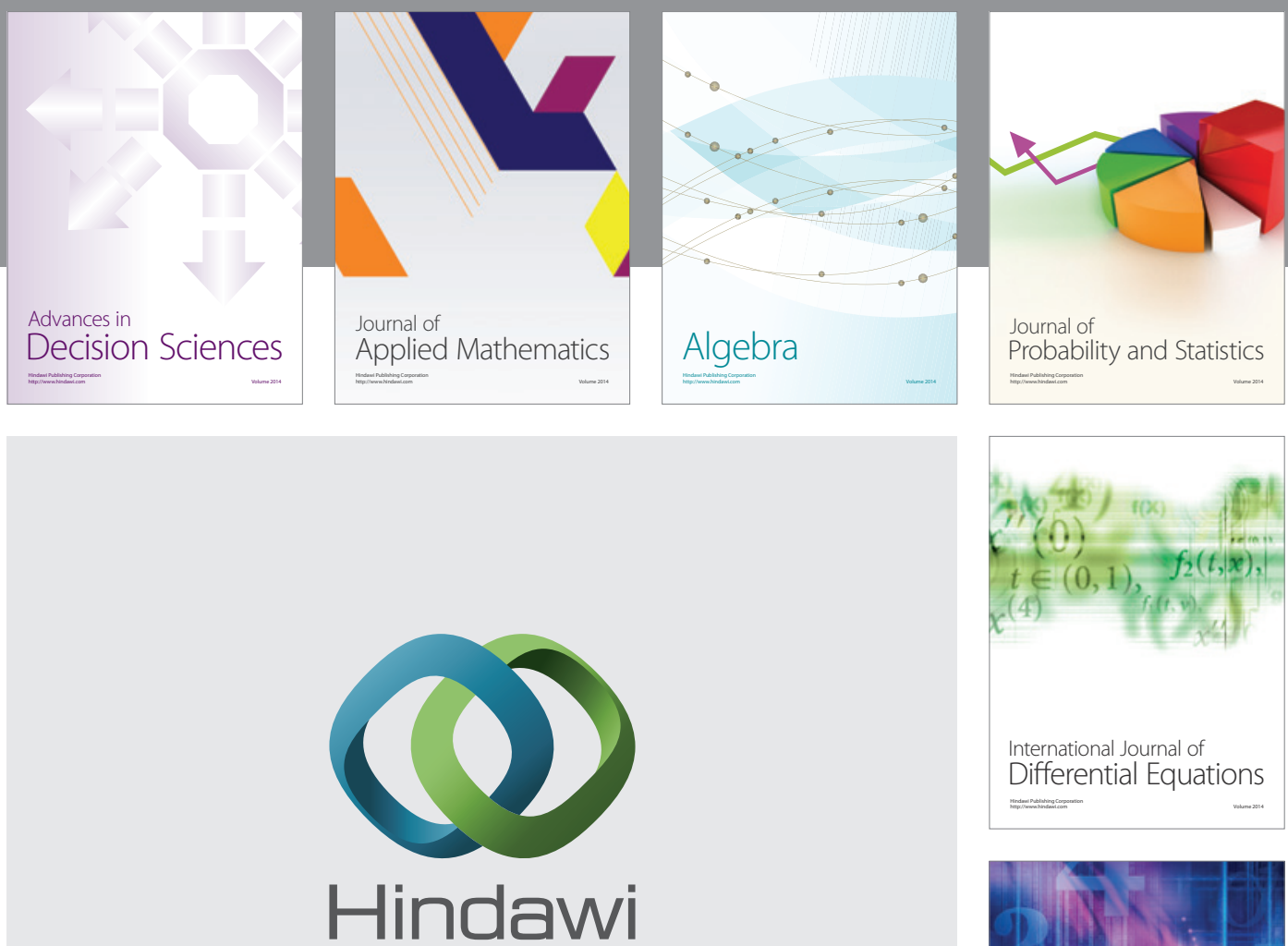

Submit your manuscripts at http://www.hindawi.com
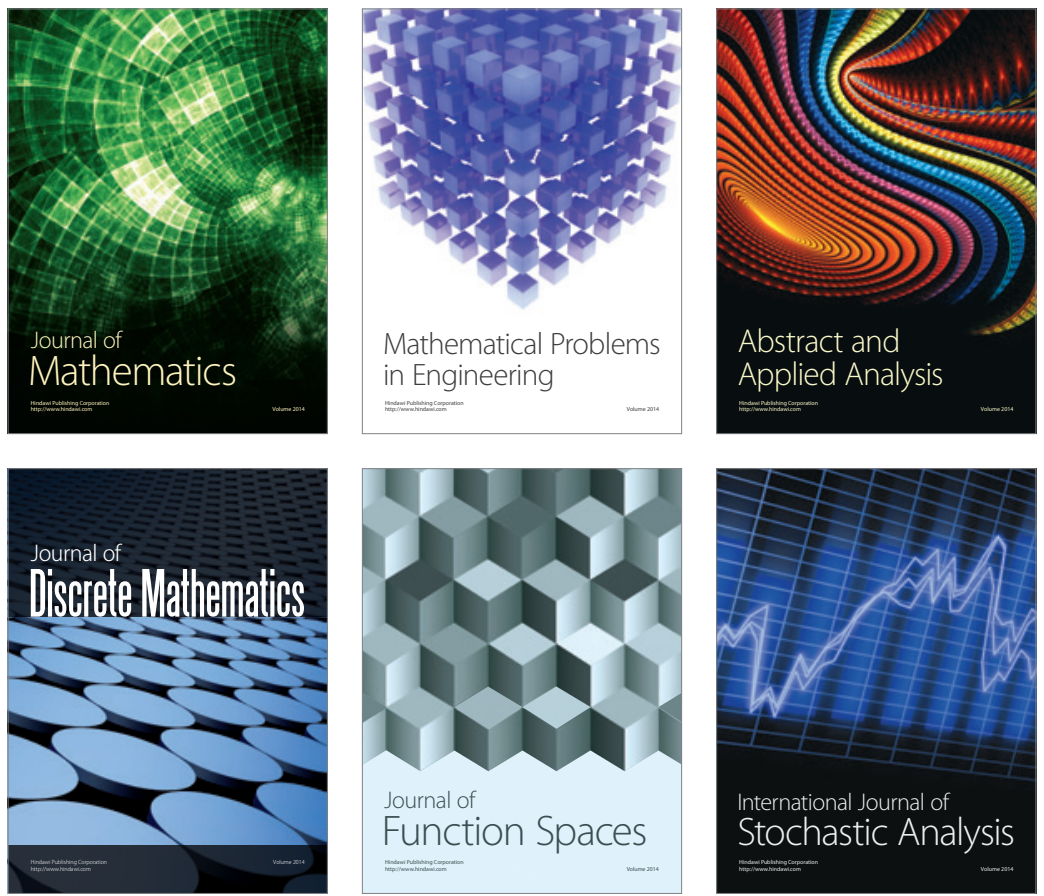

Journal of

Function Spaces

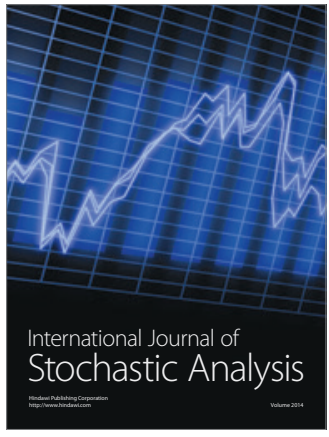

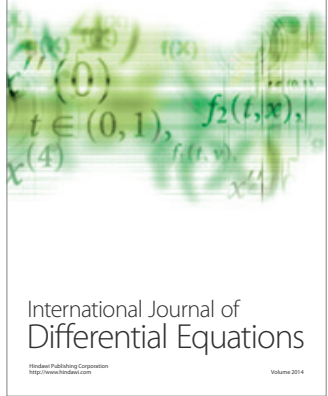
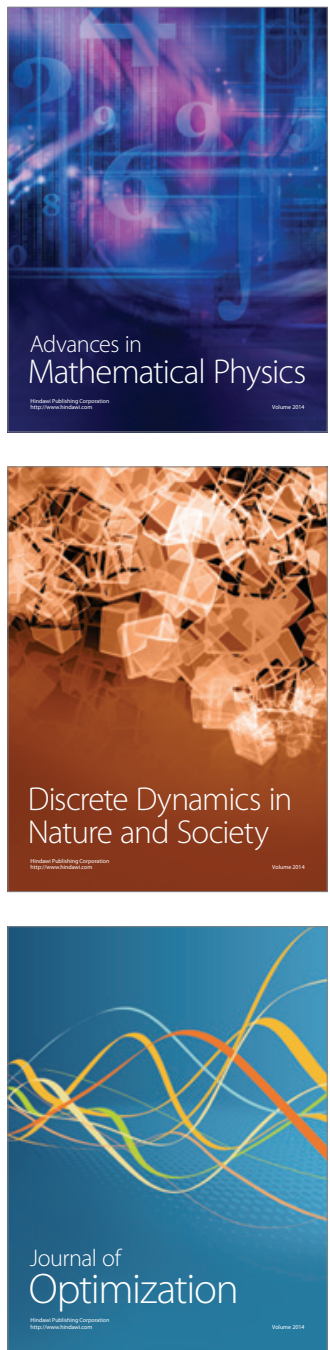